\title{
ESTUDO DA PREVALÊNCIA DE AFECÇÕES ORAIS EM EQUÍDEOS DE ARAGUAÍNA, TOCANTINS
}

\author{
Prevalence study of oral affections in equidaes of Araguaina, Tocantins \\ Estudio de la prevalencia de afecciones orales en équidos de Araguaína, Tocantins
}

\section{Haiane Arruda Luz Amorim*1, Marco Augusto Giannoccaro da Silva1, Claudia Acosta Duarte ${ }^{2}$}

${ }^{1}$ Clínica Veterinária Universitária, Curso de Medicina Veterinária, Universidade Federal do Tocantins, Araguaína, Brasil.

${ }^{2}$ Programa de Pós-graduação em Ciência Animal, Universidade Federal do Pampa, Uruguaiana, Brasil.

*Correspondência: Clínica Veterinária Universitária, Curso de Medicina Veterinária, Universidade Federal do Tocantins, BR 153, KM 112, Araguaína, Brasil.CEP:77.804-970.e-mail marcogiannoccaro@uft.edu.br.

\section{RESUMO}

Desde a domesticação, os equinos são empregados no esporte, trabalho e lazer. Para tanto, diferentes dietas foram formuladas e a utilização de embocadura para controle dos animais se fez necessária. Isso, contudo, pode induzir alterações intraorais que, além de promoverem sinais clínicos diversos, prejudicam o consumo de alimentos e o rendimento atlético. Objetivou-se com esse estudo determinar a prevalência de alterações intraorais em equinos de Araguaína, Tocantins. Foram avaliados 72 equinos por meio de exame físico geral e avaliação intraoral com espéculo. Todos os animais avaliados (100\%) apresentaram algum tipo de patologia oral. A principal alteração encontrada foi a ponta excessiva de esmalte dentário (100\%), seguida do cálculo dentário $(59,46 \%)$ e dos ganchos $(35,13 \%)$. Lesões em língua (32,43\%) e presença de "dentes de lobo" (33,78\%) também foram identificados e estes podem interferir no bem-estar animal, na mastigação e no rendimento atlético. Concluiu-se que os equinos de Araguaína possuem alterações intraorais importantes e que a avaliação oral periódica deve ser inserida nas propriedades, favorecendo a saúde animal e o desempenho satisfatório.

Palavras-chave: alterações dentárias, odontologia equina, pontas excessivas de esmalte dentário.

\section{ABSTRACT}

Since domestication, equines are employed in sports, work and recreation. For that, different diets were formulated and the use of bit to control the animals became necessary. This, however, may induce intraoral changes that, in addition to promoting various clinical signs, are detrimental to food consumption and athletic performance. The objective of this study was to determine the prevalence of intraoral alterations in horses of Araguaina, Tocantins. Seventy two horses were evaluated by means of a general physical examination and intraoral evaluation with speculum. All animals evaluated (100\%) presented some type of oral pathology. The main alteration was excessive enamel point (100\%), followed by dental calculus (59.46\%) and hooks (35.13\%). Lesions in tongue (32.43\%) and presence of "wolf teeth" (33.78\%) have also been identified and may interfere with animal welfare, chewing and athletic performance. It was concluded that the horses of Araguaina have important intraoral alterations and that the periodic oral evaluation must be inserted in the properties, favoring animal health and a satisfactory performance.

Keywords: dental disorder, equine dentistry, excessive enamel points.

\section{RESUMEN}

Desde la domesticación, los equinos son empleados en el deporte, el trabajo y paseo. Para eso, se crearon diferentes dietas y la utilización de embocaduras para control de los animales se hizo necesaria. Sin embargo, estos factores pueden inducir alteraciones orales que se manifiestan clínicamente de varias formas y perjudicar el consumo de 
alimentos y el rendimiento deportivo. Se objetivó con el presente estudio determinar la prevalencia de las alteraciones orales en caballos de Araguaína, Tocantins. Se evaluaron 72 animales por medio de lo examen físico general y la evaluación odontológica con espéculo bucal. Todos los animales evaluados (100\%) presentaban algún tipo de patología oral. La principal alteración encontrada fue la punta de esmalte (100\%) seguida del cálculo $(59,46 \%)$ y de los ganchos (35,13\%). Las lesiones en la lengua (32,43\%) y la presencia de "dientes de lobo" (33,78\%) también fueron identificados y pueden interferir en el bienestar animal, la masticación y el rendimiento deportivo. Se concluyó que los caballos de Araguaína tienen alteraciones intraorales importantes y que la evaluación oral periódica debe ser insertada en las propiedades, favoreciendo la salud animal y lo rendimiento satisfactorio.

Descriptores: alteraciones dentales, odontologia equina, puntas de esmalte.

\section{INTRODUÇÃO}

Desde a domesticação, os equinos são empregados no esporte, trabalho e lazer. Para tanto, diferentes dietas foram formuladas e a utilização de embocaduras para melhor controle do animal se fez necessária. Lane (1994), O’Neill et al., (2010) afirmaram que o desenvolvimento das anormalidades dentárias em cavalos domésticos é comum e fruto de defeitos congênitos e ou do manejo aplicado devido á domesticação.

As alterações mais frequentes são: 1) as pontas excessivas de esmalte dentário (PENCE, 2002; DIXON, 2005; RIBEIRO et al., 2011) produzidas devido a conformação da boca dos equinos, que apresenta a maxila mais larga que a mandíbula (anisognatia), aumentando o ângulo de oclusão e interferindo na biomecânica mastigatória (LANE, 1994; PETERS et al., 2006), e ao processo de domesticação, que determina alterações na dinâmica mastigatória (BAXTER, 2002), devido à alimentação com concentrados e grãos (MEDEIROS et al., 2004; DIXON, 2005; PENCE, 2002). Aparecem na face vestibular dos dentes pré-molares e molares superiores (maxilares) e na lingual dos inferiores (mandibulares). Causam, além de dificuldade mastigatória, úlceras na bochecha e língua (DIXON e DACRE, 2005); 2) os cálculos dentários, formados a partir de enfermidade renal crônica, de pouca mastigação nos casos de alimentação pobre em fibra, e pela constante exposição dos dentes caninos nos animais que possuem lábios curtos, permitindo uma secagem excessiva desses dentes (EASLEY, 2004); 3) as ondas, que aparecem quando existe o desgaste irregular da superfície oclusal, atribuída a diversos fatores, sendo: velocidade de erupção diferente, enfermidade periodontal com atraso na erupção e perda de substância dental devido a presença de cáries infundibulares (AMAYA et al., 2009); 4) os degraus, gerados pela perda de um dente, que impede o desgaste de seu contralateral, que cresce exageradamente. Restringem a mastigação, seja por algia ou impedimento mecânico, diminuindo a trituração $\mathrm{e}$, consequentemente, $\mathrm{o}$ aproveitamento adequado dos nutrientes (DIXON, 2005); 5) os ganchos, em segundo pré-molar superior ou terceiro molar inferior, resultantes do contato oclusal incompleto (DIXON e GERARD, 2006) e movimentação látero-lateral e rostro-caudal alteradas (BAKER e EASLEY, 2005; PAGLIOSA et al., 2006), prejudicam a mastigação e quando grandes, podem promover laceração na gengiva contralateral (DIXON e GERARD, 2006); 7) as rampas, que surgem como os ganchos, podem gerar pressão extrema sobre a articulação temporomandibular e criar um diastema nos segundos pré-molares inferiores, onde se acumula comida, devido à forças mecânicas geradas (JOHNSON e PORTER, 2006). Esse fato, pode levar a doença periodontal (COLLINS e DIXON, 2005; JOHNSON e PORTER, 2006). Outras alterações que podem também ser identificadas são as fraturas, prognatia e braquignatia, dentes supranumerários ou 
ausentes, defeitos de oclusão dos incisivos, desgaste transversal exagerado e cáries.

No entanto, embora se saiba que uma boa saúde oral seja fundamental para a saúde e o bem-estar animal dos equinos, ainda é reduzido e limitado o número de animais contemplados com esse cuidado no Tocantins, mais especificamente em Araguaína. Dessa forma, objetivou-se com o presente estudo identificar e determinar a prevalência de alterações intraorais em equinos de Araguaína, Tocantins.

\section{MATERIAIS E MÉTODOS}

Foram avaliados 72 animais, sendo 60 equinos e 12 muares. Os equinos estavam distribuídos da seguinte maneira: 22 da raça Quarto-de-Milha, 17 da Mangalarga Marchador, 10 da Paint Horse, 2 da Lusitano, 8 da Crioulo e 1 SRD. Os da raça Quarto-deMilha eram utilizados em provas de vaquejada, os Mangalarga Marchadores e os Muares em provas de marcha e ou trabalho na fazenda. Previamente ao exame oral, submeteu-se os animais a exame físico geral, compreendido pela determinação da frequência cardíaca e respiratória, ausculta cardíaca e dos campos pulmonares, percussão dos campos pulmonares e dos seios paranasais e auscultação dos quatro quadrantes abdominais para avaliação da motilidade intestinal.

Além disso, inspecionou-se as mucosas aparentes e os linfonodos, determinou-se o tempo de preenchimento capilar e fez-se a aferição da temperatura retal. Para auxiliar no cálculo da dosagem do fármaco utilizado para a sedação dos animais durante o exame oral, realizou-se também a pesagem dos equinos com fita de pesagem, própria para a espécie.

No procedimento anestésico utilizou-se pela via intravenosa $0,5 \mathrm{mg} / \mathrm{kg}$ de xilazina a $10 \%$ ou $0,015 \mathrm{mg} / \mathrm{kg}$ de detomidina, dependendo da disponibilidade. Após a observação dos sinais de sedação, como ptose labial, deambulação e abaixamento da cabeça, introduziu-se o abre-bocas, a cabeçada odontológica, ergueu-se a cabeça do animal e iniciou-se o exame.

A avaliação odontológica consistiu das seguintes etapas: anamnese detalhada, realizada entre a aplicação do sedativo e o início do exame, inspeção e palpação extra-oral; inspeção e palpação da arcada dentária, bochechas, língua, palato, gengivas e mucosa oral; registro das alterações encontradas em formulário individual denominado de odontograma equino. Para a identificação do dente com alteração foi utilizado o sistema de Triadan modificado.

Os dados foram tratados estatisticamente na forma de frequência absoluta (n) e relativa (\%). Não se levou em consideração o sexo, a raça, o tipo de atividade ou a alimentação.

\section{RESULTADOS E DISCUSSÃO}

$\mathrm{Na}$ Tabela 1 estão apresentadas as principais alterações orais encontradas nos equídeos de Araguaína, Tocantins. 
Tabela 1 - Frequência absoluta (FA) e Frequência Relativa (\%) das alterações observadas em equídeos de Araguaína-TO, no período de 2016 a 2017.

\begin{tabular}{c|c|c}
\hline ALTERAÇÕES & FA (n) & FR (\%) \\
\hline Cálculo Dentário & 44 & 59,46 \\
\hline Degrau & 07 & 9,46 \\
\hline Diastema & 03 & 4,05 \\
\hline Edema de Palato & 04 & 5,41 \\
\hline Fratura & 09 & 12,16 \\
\hline Gancho & 26 & 35,13 \\
\hline Lesão em Lingua & 24 & 32,43 \\
\hline Onda & 06 & 8,11 \\
\hline Pontas Excessivas de Esmalte Dentário & 72 & 100 \\
\hline Rampa & 03 & 4,05 \\
\hline
\end{tabular}

Todos os animais avaliados (72/72) no presente estudo, apresentaram algum tipo de patologia oral. A prevalência encontrada (100\%) foi superior à descrita na literatura, que varia entre $24 \mathrm{a}$ 85\% (VLAMINCK et al., 2001; PETERS et al., 2006; O’NEILL et al., 2010; BERBARI NETO et al., 2013). Provavelmente esse fato esteja relacionado a dois fatores, sendo: 1) a idade dos animais analisados, onde a grande maioria era adulto, com média de idade de 7 anos, e Baker (1979) e Du Toit et al., (2008, 2009) relacionaram o avançar da idade com o aumento da prevalência de anormalidades orais em equinos e muares; 2 ) ao tipo de alimento fornecido, onde a ração era o principal componente da alimentação de todos os animais estudados e está diretamente relacionada ao aparecimento de alterações orais, devido à mudança da mecânica mastigatória (BAXTER, 2002; CLAYTON e BONIN, 2007).

Segundo Dixon (2005), Rizzo et al., (2011), Berbari Neto et al., (2013) e Pagliosa et al., (2013) a principal alteração dentária que pode ser encontrada em equídeos é a ponta excessiva de esmalte dentário (PEED). Esta, são projeções afiadas que se formam nas bordas bucal e lingual da superfície oclusal dos dentes pré-molares e molares superiores e inferiores, respectivamente. Se dá, principalmente, por alterações na dinâmica mastigatória determinada pela domesticação (BAKER, 2002) e que causam, frequentemente, ulcerações em bochecha e língua (PAGLIOSA et al., 2006). No estudo em tela essa também foi a principal alteração observada, com prevalência de $100 \%$, corroborando com o encontrado por Hole e Dixon (2008) e de valor superior ao encontrado por Pagliosa et al., (2006), Rizzo et al., (2011) e Berbari Neto et al., (2013).

Tal achado está apoiado no fato de que a domesticação associada à mudança no manejo alimentar (adição da ração e diminuição da oferta de forragem fornecida) propicia uma diminuição no tempo de ingestão e mastigação, bem como leva a movimentos mastigatórios mais verticalizados, o que aumenta a probabilidade de ocorrência de várias alterações dentárias, inclusive a PEED (BAKER, 
2002; PAGLIOSA et al., 2006; BERBARI NETO et al., 2013).

A segunda alteração com maior prevalência foi o cálculo dentário (44/72) (59,46\%). Popularmente conhecido como tártaro e classificado como uma enfermidade intraoral adquirida, sua importância está no fato de que é um importante fator pré-disponente para o surgimento de doenças periodontais (WINTZER e AMMANN, 1990). Berbari Neto et al., (2013) registraram prevalência de $24,1 \%$ (102/423) quando analisaram cabeças de equinos em abatedouro e presumiram que esse baixo valor registrado estava relacionado ao baixo consumo de carboidrato pelos animais. Por essa razão, pode-se inferir que o resultado encontrado no estudo em tela, está pautado no elevado consumo de carboidrato pelos animais.

Ainda em relação às enfermidades adquiridas, a cárie é outra importante representante e acontece como resultado da fermentação de carboidratos por bactérias intraorais. Esse processo promove ataque ácido e descalcificação de substâncias inorgânicas do dente (DIXON, 2011b). Apenas quatro animais (5,41\%) foram diagnosticados com esta afecção, embora o carboidrato fosse o principal componente da dieta dos animais. Essa baixa ocorrência pode ser explicada pela dificuldade ou falta de experiência dos avaliadores na identificação dessa alteração.

O gancho foi a terceira alteração mais presenciada (26/74) $(35,13 \%)$ e sua ocorrência é imputada ao incompleto contato oclusal (BAKER, 2002). Lima et al., (2011) e Berbari Neto et al., (2013) encontraram valores divergentes ao deste trabalho $(65 \%$ e $28,4 \%$, respectivamente $)$ e arrolaram a presença de sobremordida e a criação extensiva como explicação para esses achados. No presente trabalho, a presença dessa alteração está diretamente ligada ao fato dos animais serem alimentados em cochos fixados acima do solo, que modifica o padrão mastigatório e beneficia o desenvolvimento dos ganchos (PAGLIOSA et al., 2006b).

Ainda, segundo Pagliosa et al., (2006b) e Dixon (2002), os locais de maior ocorrência dos ganchos são na superfície rostral dos segundos prémolares superiores (106 e 206) e na caudal dos terceiros molares inferiores (311 e 411), fato também observado nesse estudo, onde se visualizou 27 ganchos superiores e 10 inferiores.

Lesões em língua representaram 32,43\% (24/74) dos achados e estão vinculadas ao uso de embocaduras mas, principalmente, à presença de PEED nos dentes pré-molares e molares inferiores, que lesionam essa estrutura. Berbari Neto et al., (2013) detectaram em $8,7 \%$ das cabeças avaliadas, porém não relacionaram o achado a um fato específico.

Um outro fator importante a ser comentado sobre lesões em tecidos moles é a presença de edema de palato. Comumente denomina-se esse sintoma de "travagem" e diversos métodos invasivos e doloridos são utilizados para solucionar o problema. Thomassian (1990) agrega esse fato à ingestão de alimentos abrasivos e a erupção dentária e, Baker (2002), à presença das alterações de desgaste como degraus, ondas, ganchos, PEED e fraturas. Pela baixa frequência encontrada (4/74) $(5,41 \%)$ e a idade dos animais acometidos, podemos inferir que essa alteração esteja ligada primordialmente à erupção dentária.

Por fim, um dado expressivo e não inserido na Tabela 1 por não ser uma alteração propriamente dita, é a presença do "dente de lobo" (primeiro pré- 
molar) em $25(33,78 \%)$ dos 74 animais examinados. A presença desse dente é responsável por inúmeros problemas comportamentais em cavalos, causa dor oral e interferi na embocadura. Usualmente extraise esse dente quando algum tipo de sintoma é identificado (DIXON e DACRE, 2005). Como a maioria dos animais estudados estavam inseridos em alguma atividade física e muitos proprietários queixaram-se de problemas comportamentais e dificuldade na montaria, a extração desse(s) dente(s) foi indicada para que houvesse a melhoraria do desempenho atlético e promoção do bem estar animal.

\section{CONCLUSÃO}

Concluiu-se que os equinos de Araguaína possuem alterações intraorais importantes e que a avaliação oral periódica deve ser inserida nas propriedades, o que favorecerá a saúde animal e o desempenho satisfatório.

\section{AGRADECIMENTO}

Ao CNPQ, pela bolsa de Iniciação Científica concedida.

Todos os autores declararam não haver qualquer potencial conflito de interesses referente a este artigo.

\section{REFERÊNCIAS}

AMAYA, J.M.C.; VERA, L.G.; SÁNCHEZ, J. Enfermedades orales más frecuentes del caballo Criollo Colombiano. Revista CES, v. 4, n. 1, p. 4966, 2009.

BAKER, G. Dental disease in horses. In Practice 1, p. 19-26. 1979.
BAKER, G.J. Dental physiology. In: G. J. BAKER; J. EASLEY (eds.), Equine Dentistry. London, W.B. Saunders, p. 29-34; 2002.

BERBARI NETO, F.; REIBOLT, P.R.C.; DIAS, D.C.R.; NEVES, C.D.; REIS, E.M.B.; PEREIRA, G.F.P. Estudo da prevalência de afecções de cavidade oral em equídeos de matadouro. Revista Brasileira de Ciência Veterinária, v. 20, n. 4, p. 194-197, 2013.

COLLINS, N.M.; DIXON, P.M. Diagnosis and management of equine diastemata. Clinical Techniques in Equine Practice. v. 4, p. 148-154, 2005.

DIXON, P.M.; DACRE, I. A review of equine dental disorders. The Veterinary Journal, v. 169, p. 165-187, 2005.

DIXON, P.M. Acquired disorders of equine teeth. In: AAEP Proceedings - Focus Meeting, 2011.

DIXON, P.M. The gross, histological, and ultrastructural anatomy of equine teeth and their relationship to disease. In: AAEP Proceedings. v. 48, p. 421-437, 2002.

DIXON, P.M.; DACRE, I. A review of equine dental disorders. The Veterinary Journal, v. 169, p. 165-187, 2005.

DIXON, P.M.; GERARD, M.P. Oral cavity and salivary glands. In: JA Auer, JA Stick. Equine Surgery, 3ed. USA, Saunders an imprint of Elsevier, p. 321-351, 2006.

DU TOIT, N.; BURDEN, F.A.; DIXON, P.M. Clinical dental findings in 203 working donkeys in Mexico. The Veterinary Journal, v. 178, p. 380386, 2008.

DU TOIT, N.; BURDEN, F.A.; DIXON, P.M. Clinical dental examination of 357 donkeys in the UK. Part 1: Prevalence of dental disorders. Equine Veterinary Journal, v. 41, p. 390-394, 2009.

EASLEY, K.J. Equine Canine and First Premolar (Wolf) Teeth. In: 50th Annual Convention of the American Association of Equine Practitioners, Denver, CO, USA, (ed.). Publisher: American Association of Equine Practitioners, Lexington KY. Internet Publisher: International Veterinary Information Service, Ithaca NY. Diciembre 2004. 
HOLE, S.L.; DIXON, P.M. Equine dental disorders and occlusal pattern: Preliminary findings of a retrospective study (1999-2006). In: Proceedings of the British Equine Veterinary Association Congress, v. 47, p. 350, 2008.

JOHNSON, T.J.; PORTER, C.M. Infundibular Caries. In: AAEP Proceedings - Focus Meeting, 2006.

LANE, J.G. A review of dental disorders of the horse, their treatment and possible fresh approaches to management. Equine Veterinary Education, v. 6, p. 13-21, 1994.

LIMA, J.T.M.; ANDRADE, B.S.C.; SCHWARZBACH, S.V.; DE MARVAL, C.A.; LEAL, B.B.; FALEIROS, R.R.; ALVES, G.E.S. Ocorrência de doença infundibular, sobremordida e ganchos em equinos de cavalaria militar. Arquivo Brasileiro de Medicina Veterinária e Zootecnia, v. 63, n. 1 , p. 6-11, 2011.

MEDEIROS, F.; FIALHO F.; COLETTE A. Dentristia - Revisão e Descrição de 2 Casos Clínicos. O Médico Veterinário. n. 79, p. 23-40, 2004.

O'NEILL, H.V.M; KEEN, J.; DUMBELL, L. A comparison of the occurrence of common dental abnormalities in stabled and free-grazing horses. Animal, v. 4, n. 10, p. 1697-1701, 2010.

PAGLIOSA, G.M.; ALVES, G.E.S; FALEIROS, R.R.; SALIBA, E.O.S.; SAMPAIO, I.B.M.; GOMES, T.L.S.; GOBESSO, A.A.O.; FANTINI, P. Influência das pontas excessivas de esmalte dentário na digestibilidade e nutrientes de dietas de equinos. Arquivo Brasileiro de Medicina Veterinária e Zootecnia, v. 58, n. 1, p. 94-98, 2006.

PAGLIOSA, G.M.; ALVES, G.E.S.; SCHWARZBACH, S.V. Odontologia Equina Alterações dentárias frequentes que podem prejudicar a sanidade e performance do cavalo. Revista Brasileira de Medicina Equina, v. 5, n. 1, p. 7, 2006b.

PENCE, P. Equine Dentristry, a practical guide. Philadelphia. Lippincott Williams and Wilkins. 2002.

PETERS, J.W.E.; DE BOER, B.; VOORDE, G.B.M.B. Survey of common dental. In: AAEP Proceedings, v. 52, 2006.
RIBEIRO, M.G.; RIBEIRO, L.V.P; PEREIRA JUNIOR, O.C.M.; NETO, A.B.; SILVAMEIRELLES, J.R.; MEIRELLES, G.P.; PEREIRA, A.D.S. Avaliação epidemiológica e microbiológica da cavidade oral de cavalos Quarto de Milha da região Noroeste do Paraná. Revista Brasileira de Medicina Equina, v. 6, p. 4-7, 2011.

RIZZO, D.A.; LUCAS, F.A.; BARROS, R.J.S. Prevalência e diagnóstico das principais afecções dentárias de equinos da mesorregião sul do Espírito Santo. Pubvet, v. 5, n. 28, 2011.

THOMASSIAN, A. Afecções do aparelho digestivo. In: A. Thomassian (ed.), Enfermidade dos cavalos. 2 ed. São Paulo, Livraria Varela, p. 281-443, 1990.

VLAMINCK, L.; DESMET, P.; STEENHAUT, M. et al. Dental disease in the horse: a survey on 283 equine skulls. Proceedings, Tenth Annual Scientific Meeting of the European College of Veterinary Surgeons. Velbert (Germany): 2001:213-5.

WINTZER, H.J; KARL, A. Doenças dos equinos: um manual para alunos e veterinários. São Paulo: Manole, 438 p., 1990. 